\title{
Performance Measures of State Dependent MMPP/M/1 Queue
}

\author{
R.Sakthi $^{1}{ }^{*}$, V.Vidhya $^{2}$, K.Mahaboob Hassain Sherieff ${ }^{3}$ \\ ${ }^{1}$ Department of Mathematics, Saveetha School of Engineering, SIMATS, Chennai-602105 \\ ${ }^{2}$ Department of Mathematics, SAS, VIT-Chennai, India \\ ${ }^{3}$ Department of Mathematics, C. Abdul Hakeem College, Melvisharam, Vellore Dist, India \\ *Corresponding author E-mail:rsakth@gmail.com
}

\begin{abstract}
In this research work we are concerned with single unit server queue $M M P P / M / 1$ queue with Markov Modulated process in Poisson fashion and the service time follow exponential distribution. The system is framed as a state dependent with the arrival process as Markov Modulated input and service is rendered by a single server with variation in service rate based on the intensity of service state of the system. The rate matrix that is essential to compute the stationary probability vector is obtained and various performance measures are computed using matrix method.
\end{abstract}

Keywords: Single server, Markov Modulated Poisson process (MMPP), quasi-birth-death, matrix geometric method, stationary vector, performance analysis.

\section{Introduction}

Queuing systems is a mathematical phenomenon that has wide range of applications in computer systems and communication networks. It characterizes the system as the arrival, service mechanism, the number of servers and service discipline with finite or infinite service. The customer(packets) arrive to the system and wait in the buffer to be serviced, if the server fails to provide service immediately where there are many packets to be transferred that may suffer a long delay which load to poor performance. This situation can be represented by performance of queuing system with waiting length dependent on increased arrival or poor services. If the waiting length exceeds a threshold value the queue may be overloaded so that in order to reduce the queue length, the service rate may be increased.

The work carried out here is to study the state dependent quasi birth death process with MMPP inputs and the nature of problems of such special process are sometimes difficult to represent in structure. The queuing model whose work load based on dependent service rate was investigated by [3] and obtained the combined probability distribution of queue length at individual station depending on change in service rate. Some researchers $[1,5]$ suggested and proposed the revised algorithm of [8] according the model of state dependent quasi birth death process which made comfortable in matrix structure applying some analytic concepts on it. For detailed study of matrix analytic method refer $[5,8]$. Omitting the computation section of [1] the researchers distinguish clearly about the merits of their proposed algorithm with the mild approach algorithm designed by Bright and Taylor [2]. Optimal control of state-dependent service rates was investigated by Li, He \& Alfa [9] and Madan [10] were the beginners to introduce optional stages in queuing system.

In the theory of stochastic processes, the counting process has gained its importance in the field such as science, engineering etc. A variety of processes with counting nature have been familiar- ized to make the researchers some attraction towards the stochastic processes [13]. Some of the well-known counting processes are Poisson processes (PP) which is a classic in stochastic study, Markov Modulated Poisson processes (MMPP) which behaves like switch over to two different arrivals, renewal processes that repeats over a period of time etc. Markovian arrival processes (MAP) are treated as a general form of some the processes like Poisson process, Poisson processes with compound structure and modulated form of Poisson processes.

The Markov Modulated Poisson processes is often used in queuing models with modulations like on/off, high/low, priority/non priority Oliver \& Walrand [15], Feng \& Chang[4] along with that it has other interesting variety of applications in communication systems. There exists a number of papers related $[6,7,11,14,16,18,19]$, along with Scott and Smyth [18] where many processes are involved, specifically superposition of MMPPs are applied as a versatile tool to model the system like variable packet send in a traffic waiting in a long-range dependence. For a good survey on MMPP refer Fischer \& MeierHellstern [12].

In this paper we study a state dependent service waiting model in which the customer arrival is allowed to follow PP, and the system has only one server to deliver the service to the arriving customer. The description of this work as queuing model is presented in the following section.

\section{Description of the Model}

The work of this paper is about to study the model MMPP/M/1 queue with unlimited capacity. A MMPP is defined by two matrices $\mathrm{D}_{0 \mathrm{i}}$ and $\mathrm{D}_{1 i}$ with order $\mathrm{m} \times \mathrm{m}$, where $\mathrm{m}$ denotes the states of the MMPP. Let $D=D_{0 i}+D_{1 i}$ represent the generator matrix which is irreducible and infinitesimal in nature of Continuous Time Markov Chain (CTMC). The matrices $\mathrm{D}_{0 \mathrm{i}}$ and $\mathrm{D}_{1 i}$ for a two-phase MMPP are given by 
$\mathrm{D}_{0 \mathrm{i}}=\left[\begin{array}{cc}-c_{1 i} & c_{1 i} \\ c_{2 i} & -c_{2 i}\end{array}\right], \mathrm{D}_{1 \mathrm{i}}=\left[\begin{array}{cc}\lambda_{1 i} & 0 \\ 0 & \lambda_{2 i}\end{array}\right]$

where $c_{1 i}=\lambda_{1 i}+c_{11}, c_{i 2}=\lambda_{2 i}+c_{21}$ for the states $i=1,2, . . m$. The average arrival of $M M P P$ under steady state is given by $\lambda=\pi \mathrm{D} e$, where the vector $\pi$ represents the invariant state phase distribution with $\pi D=0$ and $\pi D e=1, e$ column vector that has all entries equal to 1 . An arriving customer to the system is assumed to be in random environmental state $i=1,2, \ldots, m$. The service discipline is assumed to be first come first served. The service offered by a single server with variation in service speed as its state dependent. The service rate of the server switches to different rate according to queue size $\lambda_{1}$ and $\lambda_{2}$. The rate of service is denoted as $\mu_{i}$, where $i$ is the state-owned by MMPP. The service speed $\mu_{i}$ will modulate according to the intensity of arrival. As the exponential service rate satisfies memoryless property, it is admissible that, if number of customer increase in its length the server will get intended to change its rate $\mu_{i}$ or $\mu_{i+1}$ immediately, otherwise if $n$ decreases to (n-1) the server $\mu_{i}$ will get into the change of service rate $\mu_{i-1}$.

The system state of the model assumed at time $t$ is described as bivariate process

$\{(N(t), J(t))\}$ for $t \geq 0$

Where the expression $N(t)$ represents the count of customers available in the system, $J(t)$ represents the system state of the $M M P P$ at the duration time of $t$.

The bivariate process $\{(N(t), J(t))\}$ with $t \geq 0$ represents a QBD process on the state phase $S=Z_{1} \times\{1,2, \ldots, m\}$, where $\mathrm{Z}_{1}$ is the set of non-negative integers. The infinitesimal generator representation of the QBD is given by

$\mathrm{Q}=\left(\begin{array}{ccccccc}\mathrm{B}_{00} & \mathrm{~F}_{0} & & & & & \\ \mathrm{~B}_{10} & \mathrm{~L}_{1} & \mathrm{~F}_{1} & & & & \\ & \mathrm{~B}_{2} & \mathrm{~L}_{2} & \mathrm{~F}_{2} & & & \\ & \cdots & \cdots & \cdots & & & \\ & & & & \mathrm{B}_{\mathrm{i}} & \mathrm{L}_{\mathrm{i}} & \mathrm{F}_{i} \\ & & & & \cdots & \cdots & \cdots\end{array}\right)$

where $\mathrm{B}_{00}=\left[\begin{array}{cc}-c_{00} & c_{10} \\ c_{01} & -c_{11}\end{array}\right], \mathrm{B}_{10}=d\left(\mu_{1}\right), \mathrm{B}_{\mathrm{i}}=d\left(\mu_{i}\right), \mathrm{L}_{\mathrm{i}}=$ $\mathrm{D}_{0 \mathrm{i}}-d\left(\mu_{i}\right), \mathrm{F}_{0}=\left[\begin{array}{cc}\lambda_{10} & 0 \\ 0 & \lambda_{20}\end{array}\right], \mathrm{F}_{i}=\left[\begin{array}{cc}\lambda_{1 i} & 0 \\ 0 & \lambda_{2 i}\end{array}\right]$, for $\mathrm{i}=1,2, \ldots, \mathrm{m}$.

The transition probability rate value in matrix form of this particular process is represented as given below

$\mathrm{Q}=\left(\begin{array}{cccc}\mathrm{D}_{00} & \mathrm{D}_{10} & 0 & \ldots \\ \mathrm{d}\left(\mu_{1}\right) & \mathrm{D}_{01}-\mathrm{d}\left(\mu_{1}\right) & \mathrm{D}_{11} & \ldots \\ 0 & \mathrm{~d}\left(\mu_{2}\right) & \mathrm{D}_{02}-\mathrm{d}\left(\mu_{2}\right) & \mathrm{D}_{12} \\ 0 & 0 & \mathrm{~d}\left(\mu_{3}\right) & \mathrm{D}_{03}-\mathrm{d}\left(\mu_{3}\right) \\ \cdots & \ldots & \ldots & \ldots\end{array}\right)$

Theorem: 2.1

The irreducible quasi birth death process with generator $Q$ is ergodic iff the system of equations has positive value as its solution

$\pi_{i}\left(B_{i i}+R_{i} F_{i}\right)=0$, for $i=0$

such that

$\pi_{0}\left(\sum_{i=0}^{\infty} R_{0} R_{1} \ldots R_{i-1}\right) e=1$

The components of these block matrices are given below. The element in $(j, k)$ th position of $R_{i}$, denoted by $\left[R_{i}\right]_{j, k}$ which is the average waiting time in $(i+1, k)$ th state per unit wait in $(i, j)$ th state such that it got initiated in $(i, j)$ state. The minimal non- negative rate matrix sequence $\left\{R_{i}\right\}$ play solution role to the equation set

$R^{2} B_{i}+R L_{i}+F_{i}=0, \quad 1 \leq i$.

The authors [2] proposed that shortening the series in infinite form of equations after some certain level $k$ and restate the normalizing condition in order to calculate an approximate sub-vector

$\pi_{0}(k)\left(\prod_{n=0}^{i-1} R_{i}\right)=\pi_{i}(k), \quad i=1,2, .$.

so that $\pi_{0}(k)$ satisfies the equation (1) and

$\pi_{0}(k)\left[\sum_{i=0}^{k}\left(R_{0} R_{1} \ldots R_{i-1}\right) e\right]=1$.

The vectors, $\left\{\pi_{i}(k)\right.$ : for $\left.0 \leq i\right\}$, represents an fixed probability for the limiting form of entire states or sometimes below to the level of $k$. Hence for any $k \geq 0$, when $k \rightarrow \infty$ then $\pi_{i}(k) \rightarrow \pi_{i}$ as $\pi_{i}(k)$ is an upper bound for $\pi_{i}$.

Let $H=B_{i}+L_{i}+F_{i}$, then it is readily known to $\mathrm{s} H$ is an irreducible generator. Let $\pi=\left(\pi_{1}, \pi_{2}, \ldots, \pi_{k}\right)$ be the invariant state probability vector of $H$ that satisfy the equations $\pi H=0$ and $\pi e=1$.

\section{Theorem: 2.2}

The irreducible quasi birth death process is recurrent positive iff $\boldsymbol{x} F_{i} \mathbb{1}<\boldsymbol{x} B_{i} \mathbb{1}, \boldsymbol{x} Q=\boldsymbol{x}$ and

$x \mathbb{1}=1 \quad$ for $\mathrm{i}=1,2, \ldots$

ㅁ

For more details refer Kroese[7]. Now substituting $F_{i}$ and $B_{i}$ in (5) we get $\frac{\lambda_{1 i}+\lambda_{2 i}}{\mu_{i}}<1$

\section{Performance Measures}

In this section the steady state probability vector is obtained followed by some of the valuable performances of the system in elegant manner. For the vector $\pi=\left(\pi_{1}, \pi_{2}, \ldots, \pi_{k}\right)$ assumed earlier, the stationary probability vector by matrix geometric method [14] is given by

$\pi_{n}=\pi_{1} R^{n-1}, n=1,2, \ldots$

The vectors $\pi_{i}$ are solved by the following equations

$\pi_{0} B_{00}+\pi_{1} B_{10}=0$

$\pi_{0} F_{0}+\pi_{1} L_{1}+\pi_{2} B_{2}=0$

$\pi_{1} F_{1}+\pi_{2} L_{2}+\pi_{3} B_{3}=0$

$\pi_{i} F_{i}+\pi_{i} L_{i}+\pi_{i} B_{i}=0$

$\pi_{0}+\pi_{n}(I-R)^{-1} e=1$.

In general it is tedious to calculate the exact expression of of $R$ except for some simple models. However, the approximate rate matrix of $R$ can be obtained by the following procedure

(i) $R(0)$ takes the value 0 ,

(ii) $R(n+1)=-F_{2}\left(L_{2}\right)^{-1}-R^{2}(n) B_{2}\left(L_{2}\right)^{-1}, n \geq 0$. 
The algorithm is iterative and converges, i.e $\lim _{n \rightarrow \infty} R(n)=R$ Chapter 1 [14].

The computation of performance values of the system such as the expected value of customers in the queue $L_{q}$ and system $L_{s}$, the expected waiting time of a customer waiting in the queue $W_{q}$ and system $W_{S}$, can be calculated from the invariant state probabilities The expressions for $L_{q}, L_{s}, W_{q}$ and $W_{s}$ are given by

$L_{s}=\sum_{n=1}^{\infty} n \pi_{n} \boldsymbol{e}$

$L_{q}=\sum_{n=1}^{\infty} n \pi_{n+1} \boldsymbol{e}$

$W_{s}=L_{s} / \lambda$

$W_{q}=L_{q} / \lambda$

\section{Numerical computation}

The objective of this particular part is to view the qualitative work of the queuing model considered in this study through some illustrative examples in numerical way. To accomplish the correctness accuracy of the model the results of the section 3 are computed using MATLAB code.

In this particular case problem of study, we present the impact made on the variables as well as change in the connection of the arrival with respect to change of service by computing the performance measures. We set the arrival rate varying from $\lambda_{1}=$ 0.12 to $\lambda_{2}=0.04$ and considering the varying values of $\mu_{1}$ from 1 to 2 with difference of 0.1 and $\mu_{2}$ is set as 0.7 . As the service rate increases the length of the queue resembles in the variation trend that can be observed in Figure 1 and if the length of the system changing in the trend that can be seen in Figure 2. The values are listed in the table 1 as follows

Table 1: Service rate versus Ls, $\mathrm{Lq}, \mathrm{Ws}$ and $\mathrm{Wq}$

\begin{tabular}{|c|c|c|c|c|c|}
\hline S.No & $\mu_{1}$ & Ls & $\mathrm{Lq}$ & Ws & $\mathrm{Wq}$ \\
\hline 1 & 1.1 & 0.307 & 0.0307 & 0.38375 & 0.038375 \\
\hline 2 & 1.2 & 0.271 & 0.0303 & 0.33875 & 0.037875 \\
\hline 3 & 1.3 & 0.2427 & 0.0246 & 0.303375 & 0.03075 \\
\hline 4 & 1.4 & 0.2198 & 0.0204 & 0.27475 & 0.0255 \\
\hline 5 & 1.5 & 0.201 & 0.0172 & 0.25125 & 0.0215 \\
\hline 6 & 1.6 & 0.1851 & 0.0147 & 0.231375 & 0.018375 \\
\hline 7 & 1.7 & 0.1717 & 0.0127 & 0.214625 & 0.015875 \\
\hline 8 & 1.8 & 0.16 & 0.0112 & 0.2 & 0.014 \\
\hline 9 & 1.9 & 0.1499 & 0.0098 & 0.187375 & 0.01225 \\
\hline 10 & 2.0 & 0.141 & 0.0088 & 0.17625 & 0.011 \\
\hline
\end{tabular}

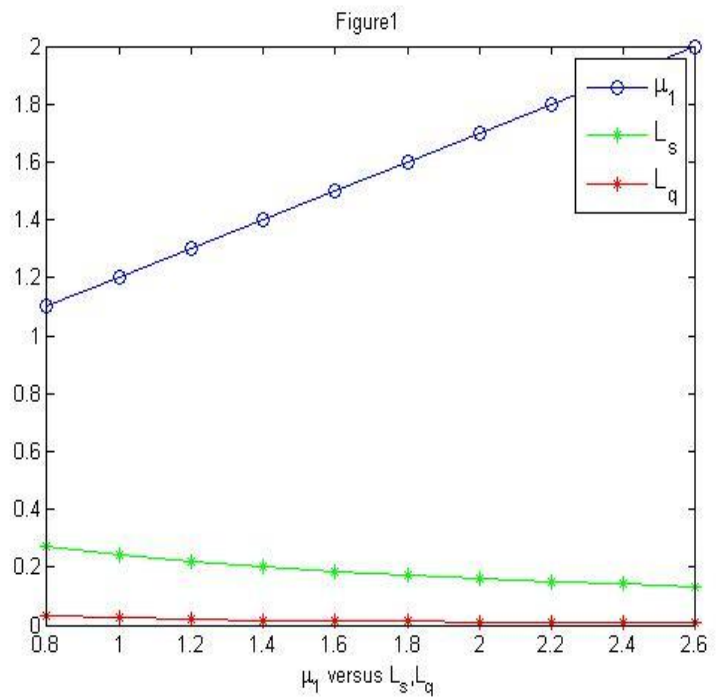

Fig 1: The graph of average arrival rate measured with average length of the system Ls and queue Lq.

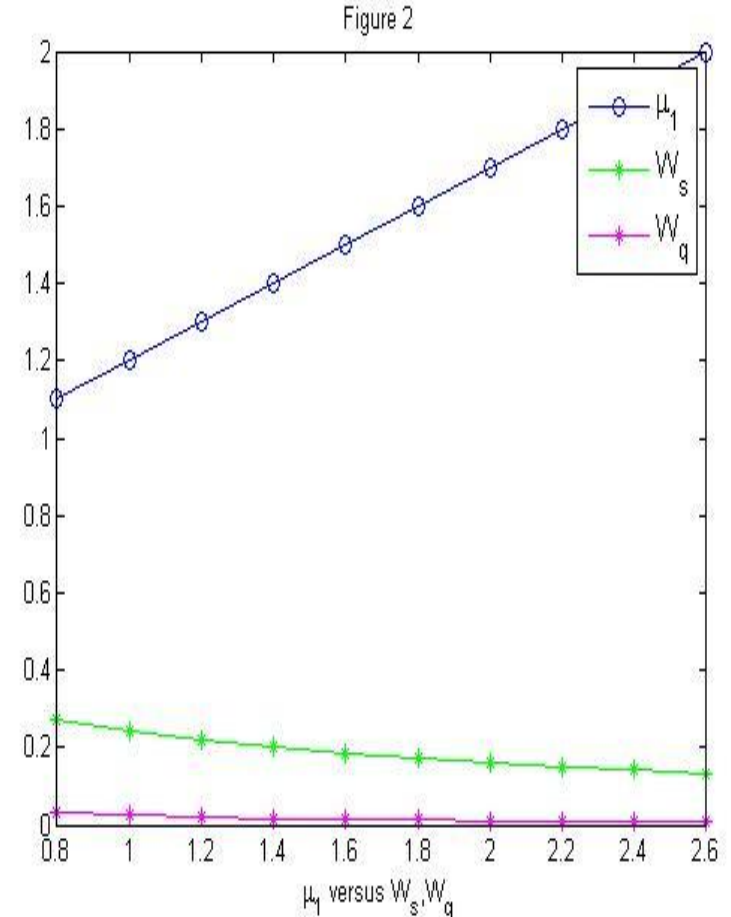

Fig 2: The graph of average arrival rate measured with average of waiting period of the system Ws and waiting period of queue Wq.

\section{Conclusion}

In this study we have described a queuing model with MMPP input with state dependent service rate. By using matrix geometric method the expression of the rate matrix which is the main ingredient to use it in the study of the qualitative behavior of queuing system is obtained along with the various performance values of the system are investigated.

\section{References}

[1] Baumann H \& Sandmann W(2010), Numerical solution of Level Dependent Quasi -Birth and Death Process, Proceedings of the International Conference on Computational Science, ICCS, Procedia Computer Science, 1: 1555-1563.

[2] Bright L \& Taylor PG (1995). Calculating the equilibrium distribution in level dependent quasi-birth-and -death process, Stochastic Models 11(3): 497-525.

[3] Delasay M, Ingolfsson A \& Kolfal B(2016), Modeling load and overwork eff ects in queuing systems with adaptive service rates, Operations Research 31

[4] Ferng HW \& Chang JF (2001), Connection wise end to end performance analysis of queuing networks with MMPP inputs, Performance Evaluation 43, 39-62.

[5] He Q.M.(2014), Fundamentals of Matrix-Analytic Methods, Springer, New York.

[6] Kharoufeh JP(2011), Level-dependent quasi-birth-and-death processes. In J. Cochran, A. Cox, P. Keskinocak, J.P. Kharoufeh, and J.C. Smith, editors, Wiley Encyclopaedia of Operations Research and Management Science, Hoboken, NJ, John Wiley \& Sons, Inc.

[7] Kroese D.P, Scheinhardt W.R.W \& Taylor P.G(2004), Spectral properties of the tandem Jackson network seen as a quasi-birth and death process, Annals of Applied Probability 14, 2057-2089.

[8] Latouche G \& Ramaswami V(1999), Introduction to Matrix Analytic Methods in Stochastic Modelling, ASA-SIAM Series on Statistics and Applied Probability, SIAM, Philadelphia, PA.

[9] Li X, He Q.M \& Alfa AS(2017), Optimal Control of StateDependent Service Rates in a MAP/M/1 Queue. IEEE Transactions of Automatic Control 62 (10), 4965 - 4979.

[10] Madan KC(2000), An M/G/1 queue with second optional service, Oueuing Systems 34, 37-48.

[11] Hellstern M.K (1989) The Analysis of a queue arising in overflow models, IEEE Trans. Communications, 37, 367-372. 
[12] Fischer W \& Hellstern M.K(1992), The Markov-modulated Poisson process (MMPP) cook book. Performance Evaluation 18(2), 149 171.

[13] Neuts M.F(1979), A versatile Markovian point process. Journal of Applied Probability, 16: 764-779.

[14] Neuts MF(1989), Matrix Geometric Solutions in Stochastic Models, John Hopkins, Baltimore, Marcel Dekker, New York.

[15] Oliver C \& Walrand J(1994), On the existence of finitedimensional filters for Markov-modulated traffic. Journal of Applied Probability 31, 515-525.

[16] Phung-Duc T, Masuyama H, Kasahara S \& Takahashi Y( 2013), A Matrix Continued Fraction Approach to Multi server Retrial Queues, Annals of Operations Research 202(1), 161-183.

[17] Ramaswami V \& Taylor PG (1996). Some properties of the rate operators in level dependent quasi-birth-and-death processes with a countable number of phases. Stoch Models 12,143-164

[18] Scott S.L \& Smyth P(2003), The Markov Modulated Poisson Process and Markov Poisson Cascade with Applications to Web Traffic Modeling. Bayesian Statistics 7. Oxford University Press.

[19] Ye J \& Li SQ (1994), Folding algorithm: A computational method for finite QBD processes with level dependent transitions. IEEE Trans. Commun 42, 625-639. 